\title{
TAX RULES TO PREVENT EXPECTATIONS-DRIVEN LIQUIDITY TRAPS
}

\author{
YoICHIRO TAMANYU \\ Keio University
}

Multiple equilibria arise in standard New Keynesian models when the nominal interest rate is set according to the Taylor rule and constrained by a zero lower bound (ZLB). One of these equilibria is deflationary and referred to as an expectations-driven liquidity trap (ELT) as it arises because of the de-anchoring of inflation expectations. This study demonstrates that a simple tax rule responding to inflation can prevent a liquidity trap from arising without increasing government spending or debt. We analytically investigate the necessary and sufficient conditions to prevent an ELT and show that both the frequency and persistence of ELT episodes affect the extent to which the tax rule must respond to inflation. In brief, the higher the frequency or the longer the persistence of the ELT, the greater the response of the tax rate must be.

Keywords: Expectations-Driven Liquidity Trap, Fiscal Policy, Monetary Policy, Regime Switching, Zero Lower Bound

\section{INTRODUCTION}

In the aftermath of the global financial crisis, more than a decade has now passed since central banks found themselves constrained by a zero lower bound (ZLB) on their policy rates. However, despite the subsequent global economic recovery, inflation has remained stubbornly low in most countries and many central banks have largely kept their policy rates virtually at zero. We often refer to situations like these where the policy rate remains stuck at the lower bound and interest rates cannot fall further as a liquidity trap (LT). Because these LTs have become a global phenomenon, investigating how the existence of the ZLB affects the economy has become a central topic in modern macroeconomics. ${ }^{1}$

Among several issues arising from the existence of the ZLB, the seminal paper by Benhabib et al. (2001) revealed that multiple equilibria emerge when the central bank targets a positive inflation rate and the nominal interest rate

The author would like to thank the associate editor, two anonymous referees, as well as Anton Braun, Fumio Hayashi, Yasuo Hirose, Daisuke Ikeda, Taisuke Nakata, and Francesco Zanetti for their helpful comments. The author acknowledges the financial support of the Keio Economic Society for the provision of English language editing. The views expressed in this paper are those of the author and do not necessarily reflect those of any affiliated institution. Address correspondence to: Yoichiro Tamanyu, 2-15-45 Mita, Minato-ku, Tokyo 108-8345, Japan; E-mail: yoichiro.tamanyu@keio.jp

(C) Cambridge University Press 2021. This is an Open Access article, distributed under the terms of the Creative Commons AttributionNonCommercial-NoDerivatives licence (https://creativecommons.org/licenses/by-nc-nd/4.0/), which permits non-commercial re-use, distribution, and reproduction in any medium, provided the original work is unaltered and is properly cited. The written permission of 
is constrained by a lower bound. Their study further showed that one of these equilibria is deflationary and the economy may become trapped in an LT without any changes in the fundamentals. In contrast to the conventional LTs triggered by large shocks to the fundamentals (fundamentals-driven LT, FLT hereafter), subsequent literature has often referred to this deflationary equilibrium as the expectations-driven LT (ELT) given it emerges solely by the de-anchoring of agents' inflation expectations.

With the prolonging of the shared experience of LTs and low inflation in many countries, the multiplicity of equilibria and the prevention of the formation of LTs have attracted a wide range of interest among academics and policymakers. Existing studies have revealed that we can prevent ELTs by the effective use of fiscal policy. For instance, Benhabib et al. (2002) propose a fiscal policy rule that responds to inflation and leads to a violation of the transversality condition if the economy were to converge to the deflationary steady state. Similarly, Schmidt (2016) demonstrates that a fiscal rule designed to keep the real marginal cost higher than a certain threshold can avoid any type of LT from arising.

However, large-scale fiscal stimulus to support the economy and the subsequent surge in government debt calls into question whether policy measures proposed in these extant studies are even plausible. This is because a policy that commits to increase government spending or debt as long as inflation remains low seems infeasible given the current situation where governments already face a huge amount of outstanding debt. More generally, policies designed to increase useless government spending only to create demand are considered unsustainable in the long run.

This paper fills this gap and contributes to the literature by demonstrating that a simple tax rule responding to inflation can prevent an ELT from arising without any increase in government spending or debt. Rather than creating demand through government expenditure, the proposed tax rule lowers the labor income tax rate and encourages households to provide more labor once the pessimistic expectations of agents prevail. Given that a simultaneous decline in both inflation and output is the key element for the ELT to arise, the inflationary pressure caused by the increase in labor supply eliminates agents' beliefs that lead to ELTs.

The analysis builds on a standard linear New Keynesian dynamic stochastic general equilibrium (DSGE) model featuring different types of shocks. To model the self-fulfilling deflation, we introduce nonfundamental "regime shocks" that force the economy to move between the targeted regime, where the inflation rate is close to its target rate, and the unintended regime, where the inflation rate is negative. In addition, we introduce shocks to the real interest rate into the model to assess how the proposed tax rule operates with conventional FLTs.

The use of a log-linearized model enables us to investigate analytically the necessary and sufficient conditions that the fiscal authority must satisfy to prevent the ELT equilibrium. We show that the frequency and persistence of the ELT episodes both affect the extent to which the tax rule must respond to inflation, such that the higher the frequency or the longer the persistence of the ELT, the 
greater the response of the tax rate must be. Nonetheless, while the recurrence of ELTs - switching between the targeted regime and the unintended regimehas important implications for policy design, extant studies have paid relatively less attention to the issue. ${ }^{2}$ Therefore, this paper contributes to the literature by showing that both the frequency and persistence of ELT episodes are crucial for policy design.

This study demonstrates that the magnitude of the changes in the tax rate lies well within a realistic range under standard calibration. We show that to prevent the ELT equilibrium, the proposed tax rule requires the fiscal authority to cut the labor income tax rate from $20 \%$ to $15 \%$ in response to a $2 \%$-point decline in annual inflation. However, we also show that if the response of the tax rate is not sufficient, the fiscal authority not only fails to prevent the ELT but also aggravates the declines in inflation and output in the ELT, relative to the case of no tax rate changes. We also consider the case where the real interest rate declines exogenously, and show that while this mitigates the decline in inflation, output is further depressed if the fiscal authority adjusts only the labor income tax rate to prevent the ELT equilibrium.

This study draws on the large literature focusing on policies to confront different types of LTs. Seminal work by Eggertsson and Woodford (2003) shows that forward guidance can mitigate the declines in inflation and output in the FLT. Subsequent studies such as Sugo and Ueda (2008) and Christiano and Takahashi (2018) also argue that monetary policy can play a central role in avoiding the ELT, while Schmitt-Grohé and Uribe (2017) find that raising the nominal interest rate to its intended target for an extended period can boost inflationary expectations and allow an escape from the ELT. These studies mainly focus on the use of monetary policy, yet several recent analyses have emphasized the importance of fiscal policy as a means to confront LTs. ${ }^{3}$

Although this paper examines the role of fiscal rules to prevent LTs, several existing studies have already explored the effectiveness of exogenous policies in an LT. Correia et al. (2013) focus on the FLT and show that appropriate tax policy can deliver stimulus without the use of government spending. Mertens and Ravn (2014) analytically consider fiscal policies in the ELT, concluding that supplyside policies, such as tax cuts, are more effective than conventional demand-side policies. Boneva et al. (2016) also show that tax cuts are effective in increasing employment in the ELT. This study shares the finding that tax cuts are effective under the ELT with these extant studies.

By comparing the economic outcomes between FLTs and ELTs under the proposed tax rule, this paper contributes to recent studies exploring effective policies under different LTs. Bilbiie (2018) compares the effects of different monetary and fiscal policies between the two LTs and shows that neo-Fisherian policy is effective in the ELT. Nakata and Schmidt (2019) provide a detailed analysis of both types of LTs, although their focus is on policymakers optimizing an assigned objective function and Cuba-Borda and Singh (2019) compare the ELT and the secular stagnation equilibrium and obtain contrasting implications for different monetary and fiscal policies. 
While the focus of this paper is to investigate theoretically the properties of ELTs, some studies empirically explore their implications. Aruoba et al. (2018) investigate whether the US and Japan have transitioned to a deflationary regime using a nonlinear DSGE model and suggest that Japan is likely to have moved to this regime in the late 1990s, whereas this is more unlikely for the USA. Hirose (2020) estimates a medium-scale DSGE model around the deflationary steady state, using Japanese data, and explores the model dynamics.

Finally, we can link this study to the literature on optimal taxation because it proposes the use of distortionary taxes to prevent LTs. It is known that tax smoothing is optimal if distortionary taxes are the only options (e.g. Barro (1979) and Lucas and Stokey (1983)). However, recent studies have found that allowing variation in tax rates can be welfare improving under certain conditions. Hagedorn (2010) studies a large class of models that feature various types of frictions and finds the conditions under which tax cycles (time-varying tax rates) are welfare improving. Arseneau and Chugh (2012) show that instead of tax smoothing, "wedge smoothing" is desirable in a model with labor market search frictions. This paper connects with this literature by showing that the effective use of distortionary taxes can improve welfare when different types of shocks disturb the economy.

The remainder of this paper is organized as follows. Section 2 details the model. Section 3 analyzes the design of tax rules to prevent the ELT equilibrium and Section 4 examines how the assumption of recurrent ELT episodes influences the results. Section 5 considers how the proposed tax rule operates in a model with fundamental shocks. Section 6 concludes.

\section{THE MODEL}

This study builds on a canonical New Keynesian DSGE model, which consists of three equilibrium equations: the downward-sloping demand equation derived from the representative household's optimization problem, the upward-sloping supply equation derived from the firm's optimization problem, and the monetary policy rule.

With the absence of the ZLB on the nominal interest rate, there are no kinks in the first two equations, and thus the equilibrium is determined uniquely. However, the existence of the ZLB generates a kink in the demand equation, which leads to multiple equilibria. Because only the most basic model is required to explore the key properties of the ELT, existing studies have largely built on this canonical three-equation model. In the following subsections, we provide the details of the model.

\subsection{Household}

A representative household gains utility from consumption and disutility from labor supply. The household maximizes its expected lifetime utility through the 
choice of consumption $c_{t}$, labor supply $l_{t}$, and bond holding $b_{t}$, given prices and subject to a budget constraint:

$$
\begin{aligned}
\max _{\left\{c_{t+s}, l_{t+s}, b_{t+s}\right\}_{s=0}^{\infty}} & \mathbb{E}_{t} \sum_{s=0}^{\infty} \beta^{s}\left[\frac{c_{t+s}^{1-\sigma}-1}{1-\sigma}-\frac{l_{t+s}^{\eta+1}-1}{\eta+1}\right], \\
\text { s.t. } & c_{t}+\frac{b_{t}}{R_{t}}=\left(1-\tau_{w, t}\right) w_{t} l_{t}+\frac{b_{t-1}}{\Pi_{t}}+d_{t},
\end{aligned}
$$

where $R_{t}$ and $\Pi_{t}$ are the gross nominal interest rate and gross inflation rate, respectively, $w_{t}$ is the real wage, $d_{t}$ is the dividend from intermediate goods firms, and $\tau_{w, t}$ is labor income tax, which is allowed to vary over time.

From the first-order conditions, we derive the Euler equation (EE) and the wage equation as

$$
\begin{aligned}
c_{t}^{-\sigma} & =\beta R_{t} \mathbb{E}_{t}\left[c_{t+1}^{-\sigma} \frac{1}{\Pi_{t+1}}\right], \\
\frac{c_{t}^{-\sigma}}{l_{t}^{\eta}} & =\frac{1}{1-\tau_{w, t}} \frac{1}{w_{t}} .
\end{aligned}
$$

The second equation shows that the labor income tax creates a wedge and affects labor supply. Simply assuming that the consumption $\left(c_{t}\right)$ and the wage $\left(w_{t}\right)$ are fixed, a lower income tax rate $\left(\downarrow \tau_{w, t}\right)$ induces the household to increase labor supply $\left(\uparrow l_{t}\right)$. This is the core mechanism through which the fiscal authority prevents the ELT equilibrium in what follows.

\subsection{Firms}

There are two types of firms in the economy: a continuum of intermediate goods producers and a final goods producer. The final goods producer uses intermediate goods as the only input and has constant elasticity of substitution production technology. The final goods producer is perfectly competitive and takes both output and input prices as given. The static profit maximization problem is as follows:

$$
\begin{array}{r}
\max _{\left\{y_{t}, y_{i, t}\right\}} P_{t} y_{t}-\int_{0}^{1} P_{i, t} y_{i, t} d i, \\
\text { s.t. } \quad y_{t}=\left(\int_{0}^{1} y_{i, t}^{\frac{\theta-1}{\theta}} d i\right)^{\frac{\theta}{\theta-1}} .
\end{array}
$$

Perfect competition drives final goods producers' profits to zero. From the firstorder conditions, we derive the demand for intermediate goods and the associated price index:

$$
\begin{aligned}
y_{i, t} & =\left(\frac{P_{i, t}}{P_{t}}\right)^{-\theta} y_{t}, \\
P_{t} & =\left(\int_{0}^{1} P_{i, t}^{1-\theta} d i\right)^{\frac{1}{1-\theta}} .
\end{aligned}
$$


There is a continuum of intermediate goods producers indexed by $i$. These producers are monopolistically competitive and incur quadratic price adjustment costs as in Rotemberg (1982). Each producer uses labor as the input in production. Firm $i$ chooses optimal price $P_{i, t}$ and labor input $l_{i, t}$ given the current aggregate output $y_{t}$ and aggregate price level $P_{t}$. It then maximizes the present value of discounted dividends after tax $d_{i, t}$ as follows:

$$
\begin{aligned}
\max _{\left\{y_{i, t+s}, P_{i, t+s}, l_{i, t+s}\right\}_{t=0}^{\infty}} & \mathbb{E}_{t} \sum_{s=0}^{\infty} Q_{c, t+s} d_{i, t+s}, \\
\text { s.t. } \quad d_{i, t+s} & =\frac{P_{i, t+s}}{P_{t+s}} y_{i, t+s}-w_{t+s} l_{i, t+s}-\frac{\psi}{2}\left(\frac{P_{i, t+s}}{P_{i, t+s-1}}-1\right)^{2} y_{t+s}, \\
y_{i, t+s} & =l_{i, t+s}, \\
y_{i, t+s} & =\left(\frac{P_{i, t+s}}{P_{t+s}}\right)^{-\theta} y_{t+s},
\end{aligned}
$$

where the real stochastic discount factor is defined as

$$
Q_{c, t+s} \equiv \beta^{s} c_{t+s}^{-\sigma} \text {. }
$$

Combining the first-order conditions and imposing symmetry across firms, we derive the Philips Curve (PC) as follows:

$$
\psi\left(\Pi_{t}-1\right) \Pi_{t}-\theta w_{t}+\theta-1=\beta \mathbb{E}_{t}\left[\frac{c_{t+1}^{-\sigma}}{c_{t}^{-\sigma}} \frac{y_{t+1}}{y_{t}} \psi\left(\Pi_{t+1}-1\right) \Pi_{t+1}\right]
$$

The aggregate production function and dividend payouts are

$$
\begin{aligned}
y_{t} & =l_{t}, \\
d_{t} & =y_{t}-w_{t} l_{t}-\frac{\psi}{2}\left(\Pi_{t}-1\right)^{2} y_{t} .
\end{aligned}
$$

\subsection{Central bank and fiscal authority}

The central bank sets the interest rate following the standard Taylor rule with the net nominal interest rate bounded from below by zero:

$$
R_{t}=\max \left[1, \frac{1}{\beta} \Pi_{t}^{\phi_{\pi}}\right]
$$

For simplicity, we assume that the interest rate responds only to inflation and not to output.

In this study, we assume that only distortionary taxes are available to finance government spending. Moreover, we allow the tax rate on labor income to vary over time. The government's budget constraint is then

$$
\frac{b_{t}}{R_{t}}+\tau_{w, t} w_{t} l_{t}=\frac{b_{t-1}}{\Pi_{t}}+g_{t}
$$


We further assume that government spending is determined endogenously. That is, the total amount of tax revenue constrains the amount of goods that the fiscal authority purchases:

$$
\tau_{w, t} w_{t} l_{t}=g_{t} .
$$

Therefore, the fiscal authority does not issue bonds in equilibrium $\left(b_{t}=0\right)$. Hence, a decrease in tax revenue results in a decrease in government spending, and this affects household income.

Most existing studies allow lump-sum transfers to isolate the marginal effect of government spending on household income. A model with a lump-sum transfer and government-spending rule nests in the results of this paper, as shown in Appendix C in Supplementary Material. Therefore, the main results of this study carry over to models that retain exogenous government spending. ${ }^{4}$

Existing studies such as Benhabib et al. (2002) and Schmidt (2016) show that fiscal rules responding to inflation can avoid the ELT. Following these findings, we assume that the labor income tax rate is a function of inflation in the following form:

$$
\tau_{w, t}=\tau_{w} \Pi_{t}^{\lambda_{w}}
$$

The fiscal authority adjusts the tax rate depending on the current inflation rate. The parameter $\lambda_{w}$ governs how aggressively the fiscal authority adjusts the tax rate in response to changes in inflation and is referred to as the "tax response parameter." If the tax response parameter is positive, the fiscal authority lowers the tax rate in response to a decline in inflation.

There have been observations in the past where tax rates have changed depending on economic conditions. For example, the Jobs and Growth Tax Relief Reconciliation Act of 2003 in the USA included cutting the tax rates on labor income and dividends, while the UK reduced its value-added tax rates throughout 2009. Although adjusting tax rates flexibly may face legislative challenges, we can view the assumption that tax rates vary depending on inflation as an extension to past examples where the adjustment in tax rates was to stimulate the economy.

Another rationale to link the tax rate with inflation lies in the fact that the current tax system is distortionary, and inflation has unequal effects on resource allocation. For example, Feldstein (1999) and Ueda (2001) estimate the welfare loss of distortionary taxation in the USA and Japan, respectively, and discuss the benefits of low inflation. While the legislative feasibility remains a challenge, Feldstein (1999) claims that indexing tax rates to inflation resolves such distortions and improves welfare.

In this study, the fiscal authority selects labor income tax as the policy instrument to influence the household's labor supply directly. As we show in the following analysis, the fiscal authority can prevent an ELT from arising by committing to lowering the labor income tax rate if the agent's pessimistic belief were to materialize and inflation to decline. Appendix B in Supplementary Material shows that the fiscal authority can prevent the ELT equilibrium by adjusting either the dividend tax or the consumption tax instead of the labor tax, while tax rates must be raised in response to a decline in inflation. 


\subsection{Equilibrium conditions}

The resource constraint of the entire economy is derived by combining equations (2), (16), and (18) as

$$
c_{t}+g_{t}+\frac{\psi}{2}\left(\Pi_{t}-1\right)^{2} y_{t}=y_{t} .
$$

Equations (3), (4), (14), (15), (17), (20), and (21) comprise the equilibrium conditions. We summarize the nonlinear equilibrium conditions other than the tax rule as the following four equations:

$$
\begin{aligned}
c_{t}^{-\sigma} & =\beta R_{t} \mathbb{E}_{t}\left[c_{t+1}^{-\sigma} \frac{1}{\Pi_{t+1}}\right], \\
\psi\left(\Pi_{t}-1\right) \Pi_{t}-\frac{\theta c_{t}^{\sigma} y_{t}^{\eta}}{1-\tau_{w, t}}+\theta-1 & =\beta \mathbb{E}_{t}\left[\frac{c_{t+1}^{-\sigma}}{c_{t}^{-\sigma}} \frac{y_{t+1}}{y_{t}} \psi\left(\Pi_{t+1}-1\right) \Pi_{t+1}\right], \\
R_{t} & =\max \left[1, \frac{1}{\beta} \Pi_{t}^{\phi_{\pi}}\right], \\
y_{t}\left[1-\frac{\psi}{2}\left(\Pi_{t}-1\right)^{2}\right] & =c_{t}+\frac{\tau_{w, t}}{1-\tau_{w, t}} c_{t}^{\sigma} y_{t}^{\eta+1} .
\end{aligned}
$$

Equilibrium conditions (22)-(25) may exhibit two different steady states. Let us call the deterministic steady state with positive inflation the "targeted steady state" and denote it by the subscript TSS. The steady-state values in the TSS are

$$
\begin{aligned}
& R_{T S S}=\frac{1}{\beta}, \\
& y_{T S S}=\left[\frac{\theta-1}{\theta}\left(1-\tau_{w}\right)\right]^{\frac{1}{\eta+\sigma}}\left[1-\tau_{w} \frac{\theta-1}{\theta}\right]^{-\frac{\sigma}{\eta+\sigma}}, \\
& c_{T S S}=\left[\frac{\theta-1}{\theta}\left(1-\tau_{w}\right)\right]^{\frac{1}{\eta+\sigma}}\left[1-\tau_{w} \frac{\theta-1}{\theta}\right]^{\frac{\eta}{\eta+\sigma}} .
\end{aligned}
$$

The following equilibrium conditions are derived by log-linearizing the tax rule (20) and the equilibrium conditions (22)-(25) around the TSS:

$$
\begin{aligned}
\hat{c}_{t} & =\mathbb{E}_{t} \hat{c}_{t+1}-\frac{1}{\sigma}\left(\hat{i}_{t}-\mathbb{E}_{t} \hat{\pi}_{t+1}\right), \\
\hat{\pi}_{t} & =\beta \mathbb{E}_{t} \hat{\pi}_{t+1}+\sigma \frac{\theta-1}{\psi} \hat{c}_{t}+\eta \frac{\theta-1}{\psi} \hat{y}_{t}-\frac{\theta-1}{\psi} \frac{\tau_{w}}{1-\tau_{w}} \hat{\tau}_{w, t}, \\
\hat{i}_{t} & =\max \left[\log \beta, \phi_{\pi} \hat{\pi}_{t}\right], \\
\hat{\tau}_{w, t} & =\lambda_{w} \hat{\pi}_{t}, \\
\gamma_{y} \hat{y}_{t} & =\gamma_{c} \hat{c}_{t}+\gamma_{\tau, w} \hat{\tau}_{w, t},
\end{aligned}
$$

where $\gamma_{y} \equiv 1-(\eta+1) \frac{\theta-1}{\theta} \tau_{w}, \quad \gamma_{c} \equiv \frac{c_{T S S}}{y_{T S S}}+\sigma \frac{\theta-1}{\theta} \tau_{w}, \quad \gamma_{\tau, w} \equiv \frac{\theta-1}{\theta} \frac{\tau_{w}}{1-\tau_{w}}$. 
In equation (31), the ZLB is imposed on the nominal interest rate after loglinearization. The variables with hats are the log deviations from the TSS values, i.e. $\hat{x}_{t} \equiv \log x_{t}-\log x_{T S S}$.

After substitution, the equilibrium conditions simplify to the following EE and PC with two variables $\hat{\pi}_{t}$ and $\hat{y}_{t}$ as

$$
\hat{y}_{t}=\xi \hat{\pi}_{t}+\mathbb{E}_{t} \hat{y}_{t+1}-\frac{1}{\sigma}\left(\max \left[\log \beta, \phi_{\pi} \hat{\pi}_{t}\right]-\mathbb{E}_{t} \hat{\pi}_{t+1}\right)-\xi \mathbb{E}_{t} \hat{\pi}_{t+1},
$$

$(1+\zeta) \hat{\pi}_{t}=\kappa \hat{y}_{t}+\beta \mathbb{E}_{t} \hat{\pi}_{t+1}$,

where $\kappa \equiv \frac{\theta-1}{\psi}\left(\eta+\sigma \frac{\gamma_{y}}{\gamma_{c}}\right), \xi \equiv \frac{\gamma_{\tau, w}}{\gamma_{y}} \lambda_{w}, \zeta \equiv \frac{\theta-1}{\psi}\left(\sigma \frac{\gamma_{\tau, w}}{\gamma_{c}}+\frac{\tau_{w}}{1-\tau_{w}}\right) \lambda_{w}$.

The log-linearized model allows us to derive closed-form solutions. Therefore, we explore effective tax rules using this model in the remainder of this paper.

Because we focus on a large fall in the real interest rate in later analysis, it is useful to define the real interest rate as follows:

$$
\begin{aligned}
\hat{y}_{t} & =\xi \hat{\pi}_{t}+\mathbb{E}_{t} \hat{y}_{t+1}-\frac{1}{\sigma}\left(\max \left[\log \beta, \phi_{\pi} \hat{\pi}_{t}\right]-\mathbb{E}_{t} \hat{\pi}_{t+1}-r_{t}^{n}\right)-\xi \mathbb{E}_{t} \hat{\pi}_{t+1}, \\
(1+\zeta) \hat{\pi}_{t} & =\kappa \hat{y}_{t}+\beta \mathbb{E}_{t} \hat{\pi}_{t+1} .
\end{aligned}
$$

The above representation is a reduced form to capture changes in the real interest rate triggered by exogenous shocks, such as changes in household preferences. The real interest rate, $r_{t}^{n}$, is an exogenous process and zero at the normal state and negative at the crisis state.

\subsection{Ensuring local determinacy around the targeted steady state}

Local indeterminacy may arise under certain parameterizations given that our proposed tax rule depends on the current inflation rate. When the ZLB does not bind, we express the equilibrium conditions (34) and (35) as the following state space representation:

$$
\begin{gathered}
{\left[\begin{array}{l}
\hat{y}_{t} \\
\hat{\pi}_{t}
\end{array}\right]=A\left[\begin{array}{c}
\mathbb{E}_{t} \hat{y}_{t+1} \\
\mathbb{E}_{t} \hat{\pi}_{t+1}
\end{array}\right],} \\
\text { where } A \equiv \frac{\sigma^{-1}}{1+\zeta+\kappa\left(\frac{\phi_{\pi}}{\sigma}-\xi\right)}\left[\begin{array}{cc}
(1+\zeta) \sigma(1+\zeta)(1-\xi \sigma)-\beta\left(\phi_{\pi}-\xi \sigma\right) \\
\kappa \sigma & \beta \sigma+\kappa(1-\xi \sigma)
\end{array}\right] .
\end{gathered}
$$

Applying the Blanchard-Kahn conditions, we derive the following condition on the tax response parameters. 
PROPOSITION 1. The log-linearized rational expectations equilibrium is locally determinate if the fiscal authority sets $\Lambda$ lower than the threshold level $\Psi^{D}$ as

$$
\begin{gathered}
\Lambda<1-\beta+\phi_{\pi} \frac{\kappa}{\sigma} \equiv \Psi^{D}, \\
\text { where } \Lambda \equiv \xi \kappa-\zeta=\frac{\theta-1}{\psi}\left(\eta \frac{\gamma_{\tau, w}}{\gamma_{y}}-\frac{\tau_{w}}{1-\tau_{w}}\right) \lambda_{w} .
\end{gathered}
$$

Proof. See Appendix A.1 in Supplementary Material.

The proposition is a generalized statement of the Taylor principle, which requires the central bank to raise the nominal interest rate more than the increase in the inflation rate. Given that the tax response parameter $\lambda_{w}$ as well as the Taylor coefficient $\phi_{\pi}$ affect the equilibrium inflation rate, both parameters must be chosen appropriately to assure local determinacy. Further, because a policy that generates indeterminacy locally around the TSS is not an appropriate option, we focus on tax rules satisfying the local determinacy condition (39) in the remaining analysis.

\subsection{Calibration}

In the model, we assume that a period corresponds to a quarter. The discount factor is set to $\beta=0.996$, which yields an annual real interest rate of $1.6 \%$. This relatively low level of real interest rate is common in recent studies on LTs. The elasticity of intertemporal substitution is chosen to be $\sigma=1$, which is a standard calibration in the literature. The Frisch elasticity of labor substitution is set to $\eta=0.4$, a value in line with the estimates by existing studies such as Boneva et al. (2016) and Smets and Wouters (2007), with parameter elasticities of 0.37 and 0.55 , respectively. The elasticity of substitution between intermediate goods is set to $\theta=6$, which yields a markup of $20 \%$. This value is consistent with the estimates of existing studies such as Broda and Weinstein (2006), which report that the median value of $\theta$ ranges from 3 to 4.3, while Denes et al. (2013) estimate $\theta$ to be approximately 13 . The price adjustment cost is set to $\psi=400$, a calibration that lies between the estimates of Ireland (2003) and Boneva et al. (2016) of 162 and 495 for the parameter, respectively.

The target net inflation rate is set equal to zero (a stable price level) and the Taylor coefficient is set to $\phi_{\pi}=1.5$, which is a standard value in New Keynesian models. The long-run labor income tax rate is set to $\tau_{w}=0.20$ following existing studies such as Mertens and Ravn (2014) and Boneva et al. (2016). These calibrations in total yield a consumption-to-output ratio of $c_{T S S} / y_{T S S}=0.83$ in the TSS.

\section{PREVENTING ELT WITH NONFUNDAMENTAL SHOCKS}

In this section, we introduce nonfundamental shocks that bring the economy into an LT without any changes in the fundamentals and characterize analytically the conditions under which the fiscal authority can prevent the ELT from arising. 


\subsection{Nonfundamental regime shocks}

Given that our primary interest is to investigate how we can design a fiscal policy to prevent an ELT, we abstract from fundamental shocks and assume that there are only two regimes in the economy: one is the "targeted regime" where inflation is near the central bank's target, while the other is the "unintended regime" where the central bank misses its inflation target and the interest rate is stuck at zero.

There is a nonfundamental shock $s_{t}$ or "regime shock" that follows a two-state Markov process. The economy is in the "targeted regime" if $s_{t}=T$ and in the "unintended regime" if $s_{t}=U$. Regime shock $s_{t}$ is revealed at the beginning of the period, which is observed by the household and firms. Private agents coordinate their decisions, and therefore their information sets when forming expectations include the current realization of $s_{t}$. The transition probability is as follows:

$$
\begin{aligned}
& \operatorname{Prob}\left(s_{t}=T \mid s_{t-1}=T\right)=p_{T}, \\
& \operatorname{Prob}\left(s_{t}=U \mid s_{t-1}=U\right)=p_{U} .
\end{aligned}
$$

Equilibrium inflation and output are denoted by $T$ in the targeted regime and $U$ in the unintended regime.

In general, the Taylor principle is not satisfied in the ELT because the nominal interest rate is constant at zero. Therefore, local indeterminacy may arise and an infinite number of equilibria can exist. One approach to handle this local indeterminacy is to introduce additional sunspot shocks as in Hirose (2020). However, because the goal of this paper is to prevent the ELT from arising and indeterminacy will not occur if it is achieved, we rule out such equilibria from our analysis.

\subsection{Equilibrium inflation and output}

We assume that the targeted regime is absorbing and impose the restriction $p_{T}=1$ on the transition probability. The advantage of an absorbing steady-state assumption is that it allows a graphical representation of the relation between the PC and the EE. This assumption is relaxed in the following section.

Whether an equilibrium exists in the unintended regime depends on the calibration. Let us first assume that we do not exclude the unintended regime from the equilibrium. In the targeted regime, the inflation rate is close to the target and the ZLB on the interest rate does not bind, while inflation is low and the interest rate remains stuck at zero in the unintended regime. We state these assumptions more formally as

$$
\begin{aligned}
& \hat{\pi}_{T} \geq \frac{\log \beta}{\phi_{\pi}} \text { and } \hat{i}_{T}=\phi_{\pi} \hat{\pi}_{T}, \\
& \hat{\pi}_{U}<\frac{\log \beta}{\phi_{\pi}} \text { and } \hat{i}_{U}=\log \beta .
\end{aligned}
$$

When the targeted regime is absorbing, equilibrium inflation and output in the targeted regime are equivalent to those in the deterministic TSS, i.e. $\hat{y}_{T}=\hat{y}_{T S S}=0$ and $\hat{\pi}_{T}=\hat{\pi}_{T S S}=0$. This indicates that the first assumption (42) is satisfied in the targeted regime. 
We obtain equilibrium inflation and output in the unintended regime by solving for the intersections of the EE and PC:

$$
\begin{aligned}
& \hat{y}_{U}=\xi \hat{\pi}_{U}-\frac{1}{\sigma} \max \left[\log \beta, \phi_{\pi} \hat{\pi}_{U}\right]+p_{U}\left(\hat{y}_{U}+\frac{1}{\sigma} \hat{\pi}_{U}-\xi \hat{\pi}_{U}\right), \\
& \hat{\pi}_{U}=\kappa \hat{y}_{U}-\zeta \hat{\pi}_{U}+\beta p_{U} \hat{\pi}_{U} .
\end{aligned}
$$

The EE divides into two sections. When the ZLB does not bind, the Taylor rule is active with the EE expressed as

$$
\hat{y}_{U}=\left[\frac{1}{\sigma} \frac{p_{U}-\phi_{\pi}}{\left(1-p_{U}\right)}+\xi\right] \hat{\pi}_{U} .
$$

Conversely, when the ZLB binds, the Taylor rule is inactive with the EE expressed as

$$
\hat{y}_{U}=-\frac{1}{\sigma} \frac{1}{\left(1-p_{U}\right)} \log \beta+\left[\frac{1}{\sigma} \frac{p_{U}}{\left(1-p_{U}\right)}+\xi\right] \hat{\pi}_{U} .
$$

Assuming that condition (43) is satisfied, inflation and output in the unintended regime can be obtained by solving equations (45) and (47) as

$$
\begin{aligned}
& \hat{\pi}_{U}=\frac{\frac{\log \beta}{1-p_{U}} \frac{\kappa}{\sigma}}{\Lambda-\left(1-\beta p_{U}\right)+\frac{p_{U}}{1-p_{U}} \frac{\kappa}{\sigma}} \\
& \hat{y}_{U}=\frac{1-p_{U} \beta+\zeta}{\kappa} \hat{\pi}_{U} .
\end{aligned}
$$

Let us consider the case where the tax rate does not respond to inflation at all $\left(\lambda_{w}=0\right)$. Figure 1 illustrates the PC and the kinked EE in the unintended regime with $p_{U}=0.92$. As shown, the EE is downward sloping in the region where the ZLB on the interest rate does not bind. In this case, the Taylor principle is satisfied and the central bank can lower the real interest rate in response to a decline in inflation. In contrast, the EE is upward sloping in the region where the ZLB binds. In this case, the central bank cannot lower its policy rate even if the inflation rate declines, which leads to an increase in the real interest rate.

The PC is upward sloping regardless of the inflation rate, which captures the standard relation that an increase in output creates upward pressure on inflation. The intersection of the PC and the upward-sloping part of the EE is the equilibrium in the unintended regime, which we refer to as the ELT equilibrium in the remainder of the paper.

Even if the tax rate does not respond to inflation at all, the ELT equilibrium may not exist under certain parameterizations. Figure 1 depicts an example in which the ELT equilibrium does not exist. We derive the condition more formally as follows.

PROPOSITION 2. Assume that the tax rate does not respond to inflation $\left(\lambda_{w}=0\right)$. The ELT equilibrium exists if and only if $p<p_{U}<1$ is satisfied 
(a)

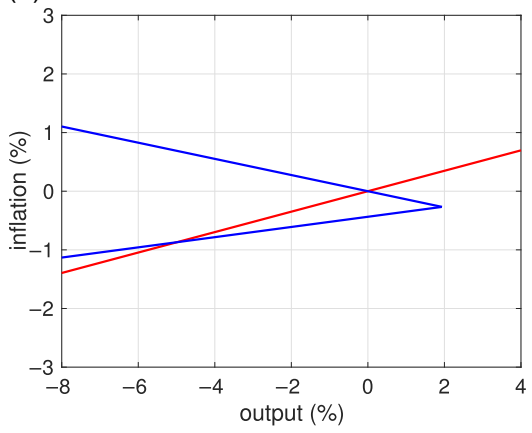

$\mathrm{EE}$ and $\mathrm{PC}$ in the unintended regime $\left(p_{U}=\right.$ 0.92 and $\left.\lambda_{w}=0\right)$. (b)

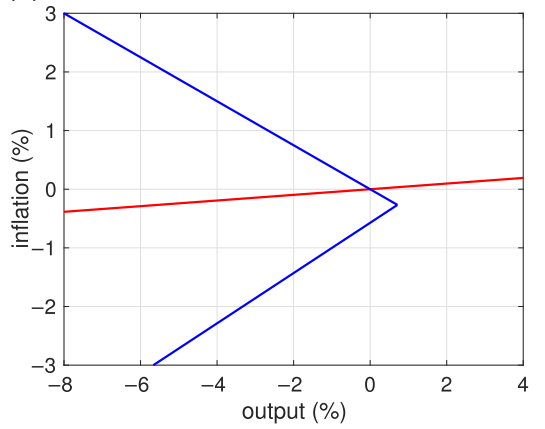

An example where the ELT does not exist $\left(p_{U}=0.70\right.$ and $\left.\lambda_{w}=0\right)$.

FigURE 1. Euler equation and Philips curve with no policy intervention.

$$
\text { where } \underline{p}=\frac{1}{2}\left(1+\frac{1}{\beta}+\frac{\kappa}{\sigma \beta}\right)-\sqrt{\frac{1}{4}\left(1+\frac{1}{\beta}+\frac{\kappa}{\sigma \beta}\right)^{2}-\frac{1}{\beta}} \text {. }
$$

Proof. See Appendix A.2 in Supplementary Material.

Proposition 2 demonstrates that the existence of the ELT equilibrium is affected by the parameterization of the transition probability $p_{U}$. As we can observe from the right-hand side of Figure 1, a simultaneous decline in both output and inflation is the key element of the ELT equilibrium. When the probability of returning to the targeted regime is high $\left(p_{U}<p\right)$ and agents expect to escape from the ELT in a relatively short period, the firm's forward-looking price-setting behavior implies a higher current inflation rate in the ELT equilibrium. Such a higher current inflation rate is not consistent with the depressed output and labor input, which contradicts the existence of the ELT equilibrium.

The prolonged experience of a zero interest rate in many advanced economies suggests that the probability of remaining in the ELT is likely to be sufficiently high. For example, Boneva et al. (2016) assume $p_{U}=0.92$ for their baseline calibration, while Aruoba et al. (2018) select $p_{U}=0.95$. Therefore, it is natural to assume that the relevant case in our study is the situation shown on the left-hand side of Figure 1, where the probability of remaining in the unintended regime is high and policy intervention is necessary to prevent the ELT equilibrium. In the remaining analysis, we set $p_{U}=0.92$ as the benchmark, which is higher than the lower bound $\underline{p} \simeq 0.89$ under our calibration.

\subsection{Preventing the ELT equilibrium by adjusting the labor income tax rate}

Let us assume that the fiscal authority chooses a suitable tax response parameter $\lambda_{w}$ to prevent the ELT equilibrium. Our previous observation that the existence 
(a)

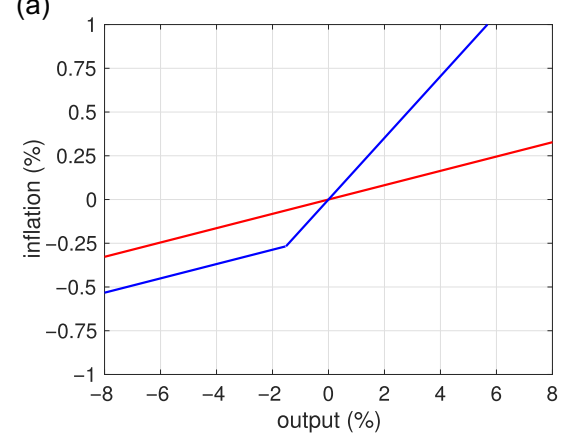

Preventing the ELT through labor income tax rate adjustment $\left(\lambda_{w}=\Psi_{w}\right)$. (b)

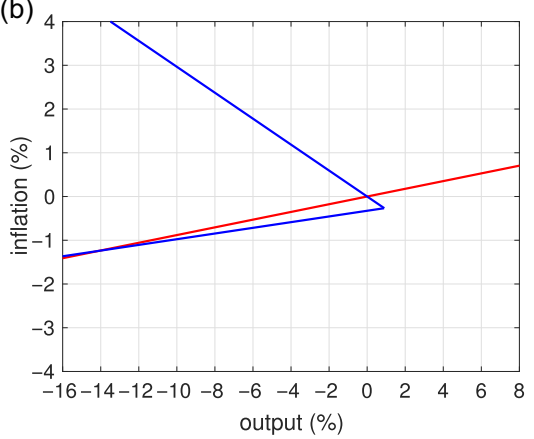

An example where the response of the tax rate is insufficient $\left(\lambda_{w}=0.3 \Psi_{w}\right)$

FIGURE 2. Euler equation and Philips curve with policy intervention.

of the ELT equilibrium depends on the model parameterization motivates us to explore whether fiscal policy can play an active role in preventing the ELT equilibrium.

PROPOSITION 3. The ELT equilibrium does not exist if and only if the fiscal authority sets $\Lambda$ equal to or lower than the threshold $\Psi$ as

$$
\Lambda \leq 1-\beta p_{U}-\frac{\kappa}{\sigma} \frac{p_{U}}{1-p_{U}} \equiv \Psi .
$$

Proof. See Appendix A.3 in Supplementary Material.

Proposition 3 demonstrates that the fiscal authority can avoid the ELT equilibrium as long as the tax rate responds sufficiently in response to changes in inflation. Condition (50) can be modified as follows:

$$
\lambda_{w} \geq-\frac{\psi}{(\theta-1) \eta}\left(\frac{1}{\eta} \frac{\tau_{w}}{1-\tau_{w}}-\frac{\gamma_{\tau, w}}{\gamma_{y}}\right)^{-1} \Psi \equiv \Psi_{w}>0 .
$$

The above inequality shows that as long as the fiscal authority sets the tax response parameter $\lambda_{w}$ equal to or higher than the threshold value $\Psi_{w}$, the ELT equilibrium does not exist.

The left-hand side of Figure 2 depicts the EE and the PC in the unintended regime when $\lambda_{w}$ is set equal to $\Psi_{w}$. The slopes of the EE and the PC are parallel in the region where the ZLB binds, which indicates that the ELT equilibrium does not exist. The partial derivative $\partial \Psi_{w} / \partial p_{U}>0$ shows that to avoid the ELT equilibrium, the fiscal authority must set the tax response parameter $\lambda_{w}$ higher as the probability of remaining in the ELT becomes higher.

How does the labor income tax rate affect the equilibrium inflation and output? As we can observe from equation (4), changes in the labor income tax rate alter the effective real wage faced by the representative household and thus change its labor supply. Provided that $\lambda_{w}$ is positive, an increase in the inflation rate raises 
the labor income tax rate, which discourages the household from supplying labor. This reduction in labor supply mitigates the inflationary pressure caused by an increase in the marginal cost and offsets the rise in inflation.

The tax rule also affects the EE because we have assumed that government spending is determined endogenously and changes in the labor income tax rate affect household income. An increase in the inflation rate leads to a rise in the labor income tax rate, and the resultant decline in the labor supply caused by the increase in the tax rate reduces household income. In the region where the ZLB does not bind, in addition to the intertemporal substitution effect caused by the increase in real interest rate, the decrease in income causes current consumption and output to decrease.

In the region where the ZLB binds, the Taylor rule is inactive and an increase in the inflation rate decreases the real interest rate, which induces the household to increase consumption through intertemporal substitution. However, the decrease in income caused by the rise in the labor income tax rate partly offsets this increase in consumption.

As shown in Appendix A.3 in Supplementary Material, $\Psi$ is always negative when $p<p_{U}<1$ is satisfied. The relation between the transition probability and the threshold level is as follows.

PROPOSITION 4. The threshold level $\Psi$ is decreasing in transition probability $p_{U}$.

Proof. See Appendix A.4 in Supplementary Material.

Thus, the greater the probability of remaining at the ELT, the more aggressively the fiscal authority must react to inflation. Namely, the fiscal authority must lower the labor income tax rate to a greater extent if the persistence of the ELT equilibrium becomes higher.

\subsection{Practical relevance of the tax rule and some caveats}

What is the magnitude of the variation in tax rate if the fiscal authority aims to prevent the ELT equilibrium? In the baseline calibration, the fiscal authority is required to set the tax response parameter to $\lambda_{w} \simeq 48$. Given that one period equals one quarter in the model, $\Delta \hat{\pi}_{t}=-0.5 \%$ is equivalent to a $2 \%$-point decline in the annual inflation rate. Because we calibrate the labor income tax rate $\tau_{w}$ to $20 \%$, the magnitude of the variation in the tax rate is well within a realistic range: that is, in response to a $2 \%$-point decline in the annual inflation rate, the fiscal authority cuts the labor income tax rate from $20 \%$ to $15 \% .^{5}$

The caveat of the proposed tax rule is that if the response parameter $\lambda_{w}$ is not sufficiently large, the fiscal authority not only fails to prevent the ELT equilibrium, but also aggravates the declines in inflation and output in the ELT. The right-hand side of Figure 2 depicts the case where the policy parameter is set to $\lambda_{w}=0.3 \Psi_{w}$, which does not satisfy the condition stated in Proposition 3. Because the tax rate does not sufficiently respond to inflation, the fiscal authority fails to eliminate 
the private sector's pessimistic expectations and the economy becomes trapped in the ELT. The inflation rate and output are then even lower than in the case where the tax rate does not respond to inflation at all.

\subsection{Connections with Schmidt (2016)}

The key findings discussed so far are closely related to those of Schmidt (2016). Both studies share the policy implication that a properly designed fiscal policy can prevent the ELT from arising by stopping real marginal cost from falling. However, some significant differences are worth noting.

First, Proposition 3 in this paper entails both the necessary and sufficient conditions to prevent the ELT, while those presented in Schmidt (2016) are mere sufficient conditions. More specifically, in the steady state, we express the nonlinear PC in equation (14) as follows:

$$
\frac{c_{S S}^{\sigma} y_{S S}^{\eta}}{1-\tau_{w, S S}}-\frac{\theta-1}{\theta}=\frac{\psi}{\theta}(1-\beta)\left(\Pi_{S S}-1\right) \Pi_{S S} .
$$

Schmidt (2016) proposes a wide class of fiscal rules that prevent ELT by violating the above nonlinear equilibrium condition (52), while the present analysis chooses a specific tax rule and derives the condition to prevent ELT by directly working with the solutions of the log-linearized model. As such, this study formally derives the relation between the tax response parameters $(\Lambda)$ and the persistence of the ELT $\left(p_{U}\right)$, and analytically shows the conditions under which the ELT equilibrium does not exist.

Second, the mechanism to prevent ELTs in Schmidt (2016) is to create demand through government expenditure; the design of the expenditure rule is such that the marginal cost of labor never declines below a certain threshold, no matter the cause of the decline. However, if financed by the lump-sum tax, the proposed fiscal rule implicitly assumes the government raises its tax collection to match expenditure. In contrast to this demand-side policy, this paper presents a supplyside policy that encourages the household to supply more labor if a self-fulfilling deflation were to emerge. We can then view the proposed rule as more favorable under the current situation where government debt has become large.

\section{PREVENTING RECURRENT ELT EPISODES}

In the previous section, we confirmed that the proposed tax rule prevents the ELT equilibrium under simplified assumptions. In this section, we relax the assumption that the unintended regime is absorbing and instead assume that the ELT is recurrent.

The recent study by Coyle and Nakata (2019) considers a model that assumes recurrent ELT episodes and finds that even a small probability of switching back to the ELT can significantly affect the optimal inflation rate. We show that while 
the qualitative results in the absorbing case carry over to the recurrent case, the fiscal authority must adjust the tax rate more compared with the absorbing case.

\subsection{Equilibrium inflation and output}

We first do not exclude the unintended regime from the equilibrium. In the targeted regime, the inflation rate is close to the target and the ZLB on the interest rate does not bind, while inflation is low and the interest rate is stuck at zero in the unintended regime. We state these assumptions formally as follows:

$$
\begin{aligned}
& \hat{\pi}_{T} \geq \frac{\log \beta}{\phi_{\pi}} \text { and } \hat{i}_{T}=\phi_{\pi} \hat{\pi}_{T}, \\
& \hat{\pi}_{U}<\frac{\log \beta}{\phi_{\pi}} \text { and } \hat{i}_{U}=\log \beta .
\end{aligned}
$$

When the ZLB does not bind as in inequality (53), the Taylor rule is active. Equilibrium conditions in the targeted regime are then

$$
\begin{aligned}
\hat{y}_{T} & =\hat{y}_{U}-\frac{1}{\sigma} \frac{\phi_{\pi}-1}{1-p_{T}} \hat{\pi}_{T}+\left(\hat{\pi}_{T}-\hat{\pi}_{U}\right)\left(\xi-\frac{1}{\sigma}\right), \\
\left(1-\beta p_{T}+\zeta\right) \hat{\pi}_{T} & =\beta\left(1-p_{T}\right) \hat{\pi}_{U}+\kappa \hat{y}_{T} .
\end{aligned}
$$

Alternatively, when the ZLB binds as in inequality (54), the Taylor rule is inactive. Equilibrium conditions in the unintended regime are then

$$
\hat{y}_{U}=\hat{y}_{T}-\frac{1}{\sigma} \frac{1}{1-p_{U}} \log \beta+\frac{1}{\sigma} \frac{1}{1-p_{U}} \hat{\pi}_{U}+\left(\hat{\pi}_{U}-\hat{\pi}_{T}\right)\left(\xi-\frac{1}{\sigma}\right),
$$

$\left(1-\beta p_{U}+\zeta\right) \hat{\pi}_{U}=\beta\left(1-p_{U}\right) \hat{\pi}_{T}+\kappa \hat{y}_{U}$.

The four equations (55)-(58) comprise the equilibrium conditions for the two regimes.

Whether the above linear system has an equilibrium satisfying both assumptions (53) and (54) depends on the model parameters. Solving equations (55)-(58), we derive the following equilibrium inflation rates for each regime:

$$
\begin{aligned}
& \hat{\pi}_{U}=\log \beta \frac{\Phi-\Omega \Lambda}{(1-\Omega) \Lambda+\Upsilon}, \\
& \hat{\pi}_{T}=\Omega\left(\hat{\pi}_{U}-\log \beta\right),
\end{aligned}
$$

where $\Omega \equiv \frac{1}{\phi_{\pi}-1} \frac{1-p_{T}}{1-p_{U}}, \quad \Phi \equiv \beta\left(1-p_{T}\right)+\frac{1-\beta p_{T}}{\phi_{\pi}-1} \frac{1-p_{T}}{1-p_{U}}+\frac{\kappa}{\sigma} \frac{1}{1-p_{U}}$

$$
\begin{aligned}
& \left(1+\frac{1-p_{T}}{\phi_{\pi}-1}\right), \Upsilon \equiv \frac{\kappa}{\sigma} \frac{1}{1-p_{U}}\left(p_{U}+\frac{1-p_{T}}{\phi_{\pi}-1}\right)-\beta\left(1-p_{T}\right)+\frac{1-\beta p_{T}}{\phi_{\pi}-1} \frac{1-p_{T}}{1-p_{U}} \\
& -\left(1-\beta p_{U}\right)+\frac{\beta\left(1-p_{T}\right)}{\phi_{\pi}-1} .
\end{aligned}
$$


Solutions (59) and (60) show that when both regimes are recurrent, the equilibrium inflation rate is affected by both transition probabilities $p_{T}$ and $p_{U}$.

\subsection{Conditions to prevent recurrent ELTs}

Let us first assume that the tax rate does not respond to inflation $\left(\lambda_{w}=0\right)$. We can then derive the following condition under which the ELT equilibrium exists.

PROPOSITION 5. Assume that the tax rate does not respond to inflation $\left(\lambda_{w}=0\right)$. The ELT equilibrium exists if and only if $\Phi \phi_{\pi}>\Upsilon>0$ is satisfied.

Proof. See Appendix A.5 in Supplementary Material.

The above condition is analogous to that in Proposition 2, which claims that the ELT equilibrium exists only under a certain combination of parameters. In particular, the existence of the ELT equilibrium depends on both probabilities $p_{T}$ and $p_{U}$.

We can derive the condition under which the fiscal authority prevents the ELT.

PROPOSITION 6. The ELT equilibrium does not exist if and only if the fiscal authority sets $\Lambda$ equal to or lower than the threshold $\tilde{\Psi}$ as

$$
\Lambda \leq-\frac{\Upsilon}{1-\Omega} \equiv \tilde{\Psi}
$$

Proof. See Appendix A.6 in Supplementary Material.

The condition can be modified as

$$
\lambda_{w} \geq-\frac{\psi}{(\theta-1) \eta}\left(\frac{1}{\eta} \frac{\tau_{w}}{1-\tau_{w}}-\frac{\gamma_{\tau, w}}{\gamma_{y}}\right)^{-1} \tilde{\Psi} \equiv \tilde{\Psi}_{w}>0 .
$$

Taking the derivative of $\tilde{\Psi}$ with respect to transition probabilities $p_{U}$ and $p_{T}$, we obtain the following two relations:

$$
\begin{aligned}
& \frac{\partial \tilde{\Psi}}{\partial p_{U}}=-\beta-\frac{\kappa}{\sigma} \frac{1}{\left(p_{U}-1+\frac{1-p_{T}}{\phi_{\pi}-1}\right)^{2}}, \\
& \frac{\partial \tilde{\Psi}}{\partial p_{T}}=-\beta+\frac{\kappa}{\sigma} \frac{\phi_{\pi}-1}{\left[1-p_{T}+\left(p_{U}-1\right)\left(\phi_{\pi}-1\right)\right]^{2}} .
\end{aligned}
$$

The former derivative (63) is negative for any combination of $p_{U}$ and $p_{T}$, which indicates that the fiscal authority must set $\lambda_{w}$ larger as the persistence of the unintended regime becomes longer.

The latter derivative (64) is positive when the following inequality is satisfied:

$$
p_{T}>1+\left(p_{U}-1\right)\left(\phi_{\pi}-1\right) \equiv \underline{p}_{T} .
$$

Under the benchmark calibration of $p_{U}=0.92$, the cutoff value is $\underline{p}_{T}=0.96$. The inequality shows that the fiscal policy needs to respond to inflation more as the probability of switching back to the unintended regime $\left(1-p_{T}\right)$ becomes higher. 


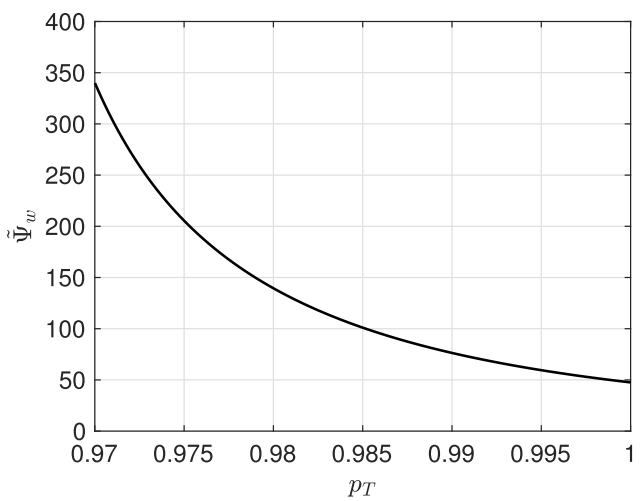

FIGURE 3. Threshold value $\tilde{\Psi}_{w}$ under different transition probabilities $p_{T}$.

Figure 3 shows how the threshold $\tilde{\Psi}_{w}$ changes depending on the transition probability $p_{T}$. Given $\tilde{\Psi}_{w} \rightarrow \Psi_{w}$ holds as $p_{T} \rightarrow 1$, the qualitative results in the absorbing case carry over to the case of recurrent ELT episodes. Besides, because $p_{T}$ is close to one, the size of the tax response parameter is little affected by the recurrence of the ELT equilibrium. For example, $p_{T}=0.99$ is associated with the tax response parameter of $\tilde{\Psi}_{w} \simeq 76$. However, as the probability of switching back to the unintended regime becomes higher and approaches the bifurcation point $p_{T} \rightarrow \underline{p}_{T}, \tilde{\Psi}_{w}$ increases significantly.

Why do both the frequency and persistence of the unintended regime matter? Intuitively, both a higher frequency $\left(1-p_{T}\right)$ and a longer persistence $\left(p_{U}\right)$ increase the average duration of remaining in the unintended regime. As the duration becomes longer, agents come to believe that expected inflation remains low on average even in the intended regime. In such a case, a modest drop in current output and inflation suffices for the self-fulfilling deflation to materialize; the fiscal authority should not tolerate even a modest decline in inflation to avoid the ELT. To eliminate such deflationary expectations, the authority must commit to lowering the labor income tax rate more in response to a decline in inflation.

\section{THE ROLE OF TAX RULES WITH FUNDAMENTAL SHOCKS}

In this section, we examine how the inflation-sensitive tax rule performs in the stylized model with fundamental shocks. We show that the proposed tax rule can mitigate the declines in inflation in an LT triggered by a severe fall in the real interest rate, while output falls further if only the labor income tax rate adjusts.

\subsection{Real interest rate shocks}

Following the existing studies on FLTs such as Eggertsson and Woodford (2003), we assume that the real interest rate $r_{t}^{n}$ is stochastic. ${ }^{6}$ Let us consider a two-state 
Markov model where the real interest rate takes $r_{t}^{n}=r_{H}^{n}$ in the "normal state" and $r_{t}^{n}=r_{L}^{n}$ in the "crisis state." The transition probability is

$$
\begin{aligned}
& \operatorname{Prob}\left(r_{t}^{n}=r_{H}^{n} \mid r_{t-1}^{n}=r_{H}^{n}\right)=p_{H}^{*}, \\
& \operatorname{Prob}\left(r_{t}^{n}=r_{L}^{n} \mid r_{t-1}^{n}=r_{L}^{n}\right)=p_{L}^{*} .
\end{aligned}
$$

To facilitate comparability with existing work, let us assume that the normal state is absorbing and $p_{H}^{*}=1$ in the remaining analysis.

\subsection{Equilibrium inflation and output}

When the normal state is absorbing, inflation and output in the normal state are equivalent to those in the targeted deterministic steady state, i.e. $\hat{y}_{H}=\hat{y}_{T S S}=0$ and $\hat{\pi}_{H}=\hat{\pi}_{T S S}=0$. The real interest rate in the normal state is $r_{H}^{n}=0$.

Equilibrium inflation and output in the crisis state can be obtained by solving for the intersections of the $\mathrm{EE}$ and the $\mathrm{PC}$ with the real interest rate set to $r_{t}^{n}=r_{L}^{n}$ as

$$
\begin{aligned}
& \hat{y}_{L}=-\frac{1}{\sigma} \max \left[\log \beta, \phi_{\pi} \hat{\pi}_{L}\right]+\frac{1}{\sigma} r_{L}^{n}+\xi \hat{\pi}_{L}+p_{L}^{*}\left[\hat{y}_{L}+\frac{1}{\sigma} \hat{\pi}_{L}-\xi \hat{\pi}_{L}\right], \\
& \hat{\pi}_{L}=p_{L}^{*} \beta \hat{\pi}_{L}+\kappa \hat{y}_{L}-\zeta \hat{\pi}_{L} .
\end{aligned}
$$

Let us assume that the fall in the real interest rate is sufficiently large and $r_{L}^{n}=-0.01<\log \beta$.

Whether an equilibrium exists in the crisis state depends on the parameterization. Let us first assume that an equilibrium exists in the crisis state. Following similar steps to the case with regime shocks, inflation and output in the crisis state can be solved as

$$
\hat{\pi}_{L}=\left\{\begin{array}{l}
\frac{-r_{L}^{n} \frac{\kappa}{1-p_{L}^{*}} \frac{\sigma}{\sigma}}{\Lambda-\left(1-\beta p_{L}^{*}\right)-\frac{\phi_{\pi}-p_{L}^{*}}{1-p_{L}^{*}} \frac{\kappa}{\sigma}} \text { if } \hat{\pi}_{L} \geq \frac{\log \beta}{\phi_{\pi}} \\
\frac{\log \beta-r_{L}^{n} \frac{\kappa}{\sigma}}{\Lambda-\left(1-\beta p_{L}^{*}\right)+\frac{p_{L}^{*}}{1-p_{L}^{*}} \frac{\kappa}{\sigma}} \text { if } \hat{\pi}_{L}<\frac{\log \beta}{\phi_{\pi}}
\end{array},\right.
$$

The above solution shows that whether the ZLB binds in the crisis state depends on the choice of the parameters.

Let us first consider the case where the tax rate does not respond to inflation $\left(\lambda_{w}=0\right)$. Similar to the case with regime shocks, we can establish the following proposition. 
PROPOSITION 7. Assume that the tax rate does not respond to inflation $\left(\lambda_{w}=0\right)$. An equilibrium exists in the crisis state if and only if $0<p_{L}^{*}<\underline{p}$. In particular, the equilibrium exhibits an LT if and only if $p_{\dagger}<p_{L}^{*}<\underline{p}$

where $p_{\dagger}=\frac{1}{2}\left(1+\frac{1}{\beta}+\frac{\kappa}{\sigma \beta}\right)-\sqrt{\frac{1}{4}\left(1+\frac{1}{\beta}+\frac{\kappa}{\sigma \beta}\right)^{2}-\frac{1}{\beta}-\frac{\kappa}{\sigma \beta} \phi_{\pi}+\frac{\kappa}{\sigma \beta} \frac{\phi_{\pi}}{\log \beta} r_{L}^{n}}$.

Proof. See Appendix A.7 in Supplementary Material.

Proposition 7 asserts that $p_{L}^{*}$ cannot be too high for the equilibrium to exist in the crisis state: this is a feature often pointed out in the literature. Note that for the equilibrium in both the crisis state and the unintended regime to exist, $p_{L}^{*}<p<p_{U}$ must be satisfied. If the decline in the real interest rate is relatively short lived $\left(0<p_{L}^{*} \leq p_{\dagger}\right)$, the ZLB may not bind in the crisis state. Given that our primary interest is to explore policies in the LT, we rule out such occasions and restrict our focus to the case where ZLB binds in the crisis state $\left(p_{\dagger}<p_{L}^{*}<p\right)$. As a benchmark, we set the probability of remaining in the crisis state to $p_{L}^{*}=0.85$, which is between the lower bound $p_{\dagger} \simeq 0.79$ and the upper bound $\underline{p} \simeq 0.89$ under our calibration.

Next, let us assume that the fiscal authority makes a suitable choice on the tax response parameter to avoid the ELT. The following proposition formally establishes the effect of the inflation-sensitive tax rule on equilibrium inflation in the crisis state.

PROPOSITION 8. If the fiscal authority targets in such a way as to avoid the ELT, the inflation rate in the crisis state is always higher than where the tax rate does not respond to inflation at all.

Proof. See Appendix A.8 in Supplementary Material.

Figure 4 illustrates the EE and the PC in the crisis state with different tax response parameters. The dotted lines denote the case of $\lambda_{w}=0$. In this case, the inflation rate is significantly low and output depressed in the crisis state. The solid lines show the EE and the PC with the response parameters set to $\lambda_{w}=\Psi_{w}$, where $\Psi_{w}$ is chosen to avoid the ELT with the calibration $p_{U}=0.92$. Indeed, inflation is higher than the case of no policy intervention in this case. However, the output is significantly depressed in the crisis state with an intervention because a cut in the labor income tax is associated with a decrease in government spending, which leads to a further decrease in aggregate demand.

The above observation shows that to mitigate the decline in both output and inflation in the crisis state, targeting a single tax rate is not sufficient. Similar shortcomings have been pointed out by recent studies that explore effective policies under different LTs. Bilbiie (2018) summarizes the differences in the policy effects between the two LTs and shows that a temporary tax cut improves welfare in the ELT while its impact is negative in the FLT, which is close to our finding. 


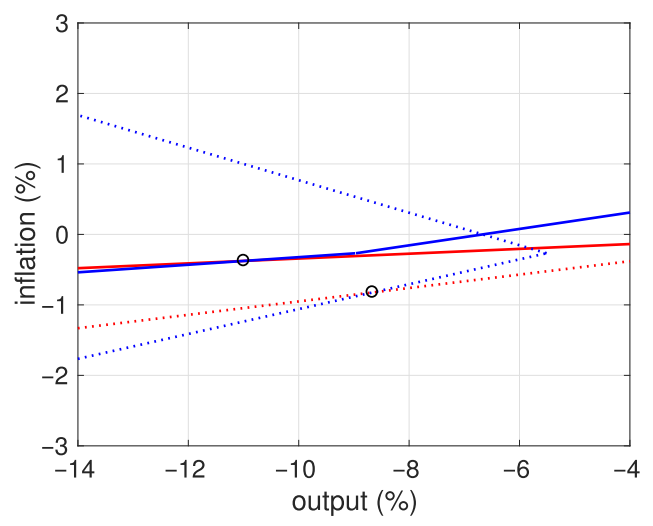

Note: Dotted lines represent EE and PC with $\lambda_{w}=0$ and the solid lines EE and PC with $\lambda_{w}=\Psi_{w}$. Black circles indicate the intersections.

FIGURE 4. Euler equation and Philips curve in the crisis state.

Cuba-Borda and Singh (2019) show that none of the major policy measuresgovernment spending, supply shocks, and neo-Fisherian policies-are effective for both types of LTs, while a minimum wage policy can prevent both of the LTs.

In Appendix B in Supplementary Material, we show that the fiscal authority can achieve both higher output and inflation in the crisis state if it combines different tax instruments appropriately. More concretely, fiscal policy can prevent the ELT equilibrium and improve welfare in the crisis state by affecting both household consumption demand and labor supply. However, the fiscal authority must lower the labor income tax rate on one hand and raise the dividend (or consumption) tax rate on the other hand to improve both inflation and output.

\section{CONCLUSION}

In this paper, we showed that a simple tax rule that responds to inflation could prevent an economy from falling into an ELT, and investigated analytically the conditions under which the proposed tax rule can prevent the ELT. The study also investigated the effect on the fiscal rule when the ELT episodes are recurrent.

While this paper shows that the proposed tax rule can improve allocations in ELTs, we have abstracted from other potentially relevant disturbances that may affect the welfare, such as cost-push and policy shocks. Given that this study relies on the use of distortionary taxes, desirable policies may differ when alternative shocks impact the economy.

The primary mechanism through which the fiscal authority prevents the ELT is by inducing the household to increase its labor supply when the inflation rate declines. Recent studies such as Schmitt-Grohé and Uribe (2017) and Cuba-Borda and Singh (2019) assume inelastic labor supply. Under such cases, a minimum wage policy that installs a lower bound on deflation can be more effective than that affecting the marginal behavior of the household. Introducing other relevant 
frictions such as downward nominal wage rigidity may also affect our results. We defer such an in-depth investigation with different assumptions to future work.

\section{SUPPLEMENTARY MATERIAL}

To view supplementary material for this article, please visit http://doi.org/ 10.1017/S136510052000067X.

\section{NOTES}

1. For recent developments in the literature exploring how the ZLB affects the economy, see Debortoli et al. (2019), Liu et al. (2019), and Ikeda et al. (2020) to mention a few.

2. Coyle and Nakata (2019) show that the optimal inflation rate changes significantly when we assume ELT episodes to be recurrent.

3. Investigating the effectiveness of fiscal policy at the ZLB is an active research area. For recent developments, see Bilbiie et al. (2019), Ercolani and Valle e Azevedo (2019), and Ngo (2019), among many others.

4. Endogenous government spending is motivated by the fact that a lump-sum transfer is not available in reality. When the fiscal authority increases (or at least maintains) its expenditure while its tax revenue decreases due to depressed economic activity, the fiscal authority must increase bond issuance. This decrease in tax revenue and an increase in expenditure have led to the current elevated government debt. By assuming endogenous government spending, the fiscal authority allows variation in the tax revenue and avoids the increase in government debt. However, the mechanism through which the fiscal authority prevents the ELT does not rely on this endogenous government-spending assumption, as shown in Appendix C in Supplementary Material.

5. The result is comparable to the magnitude of the tax variation proposed in Correia et al. (2013). In their benchmark case, consumption taxes increase from 5\% to $14 \%$ and labor income taxes decrease from $28 \%$ to $21 \%$ to counteract the shock in the discount factor. Note that they also include an investment tax credit in their model, which jumps in the first period to $9 \%$ and then decreases gradually toward 0 .

6. In our model, $r_{t}^{n}$ is expressed as the deviation from the steady-state gross real interest rate $R^{n}=1 / \beta$.

\section{REFERENCES}

Arseneau, D. M. and S. K. Chugh (2012) Tax smoothing in frictional labor markets. Journal of Political Economy 120(5), 926-985.

Aruoba, B., P. Cuba-Borda, and F. Schorfheide (2018) Macroeconomic dynamics near the ZLB: A tale of two countries. Review of Economic Studies 85, 87-118.

Barro, R. (1979) On the determination of the public debt. Journal of Political Economy 87(5), 940-971.

Benhabib, J., S. Schmitt-Grohé, and M. Uribe (2001) The perils of taylor rules. Journal of Economic Theory 96, 40-69.

Benhabib, J., S. Schmitt-Grohé, and M. Uribe (2002) Avoiding liquidity traps. Journal of Political Economy 110(3), 535-563.

Bilbiie, F. O. (2018) Neo-Fisherian policies and liquidity traps. CEPR Discussion Papers 13334, C.E.P.R. Discussion Papers.

Bilbiie, F. O., T. Monacelli, and R. Perotti (2019) Is government spending at the zero lower bound desirable? American Economic Journal: Macroeconomics 11(3), 147-173.

Boneva, L. M., R. A. Braun, and Y. Waki (2016) Some unpleasant properties of loglinearized solutions when the nominal rate is zero. Journal of Monetary Economics 84, 216-232. 
Broda, C. and D. E. Weinstein (2006) Globalization and the gains from variety. The Quarterly Journal of Economics 121(2), 541-585.

Christiano, L. and Y. Takahashi (2018) Discouraging deviant behaviors in monetary economics. mimeo.

Correia, I., E. Farhi, J. P. Nicolini, and P. Teles (2013) Unconventional fiscal policy at the zero bound. American Economic Review 103(4), 1172-1211.

Coyle, P. and T. Nakata (2019) Optimal inflation target with expectations-driven liquidity traps. Finance and Economics Discussion Series 2019-036, Board of Governors of the Federal Reserve System.

Cuba-Borda, P. and S. R. Singh (2019) Understanding persistent stagnation. International Finance Discussion Papers 1243, Board of Governors of the Federal Reserve System.

Debortoli, D., J. Galí, and L. Gambetti (2019) On the empirical (ir)relevance of the zero lower bound constraint. In NBER Macroeconomics Annual 2019, volume 34, NBER Chapters, pp. 141-170. National Bureau of Economic Research, Inc.

Denes, M., G. B. Eggertsson, and S. Gilbukh (2013) Deficits, public debt dynamics and tax and spending multipliers. The Economic Journal 123(566), F133-F163.

Eggertsson, G. B. and M. Woodford (2003) The zero bound on interest rates and optimal monetary policy. Brookings Papers on Economic Activity 1, 139-233.

Ercolani, V. and J. Valle e Azevedo (2019) How can the government spending multiplier be small at the zero lower bound? Macroeconomic Dynamics 23(8), 3457-3482.

Feldstein, M. S. (1999) Capital income taxes and the benefit of price stability. In The Costs and Benefits of Price Stability, NBER Chapters, pp. 9-46. National Bureau of Economic Research, Inc.

Hagedorn, M. (2010) Ramsey tax cycles. The Review of Economic Studies 77(3), 1042-1071.

Hirose, Y. (2020) An estimated DSGE model with a deflation steady state. Macroeconomic Dynamics 24(5), 1151-1185.

Ikeda, D., S. Li, S. Mavroeidis, and F. Zanetti (2020) Testing the effectiveness of unconventional monetary policy in Japan and the United States. IMES Discussion Paper Series 20-E-10, Institute for Monetary and Economic Studies, Bank of Japan.

Ireland, P. N. (2003) Endogenous money or sticky prices? Journal of Monetary Economics 50(8), 1623-1648.

Liu, P., K. Theodoridis, H. Mumtaz, and F. Zanetti (2019) Changing macroeconomic dynamics at the zero lower bound. Journal of Business \& Economic Statistics 37(3), 391-404.

Lucas, R. and N. Stokey (1983) Optimal fiscal and monetary policy in an economy without capital. Journal of Monetary Economics 12(1), 55-93.

Mertens, K. R. S. M. and M. O. Ravn (2014) Fiscal policy in an expectations-driven liquidity trap. Review of Economic Studies 81(4), 1637-1667.

Nakata, T. and S. Schmidt (2019) Expectations-driven liquidity traps: implications for monetary and fiscal policy. Working Paper Series 2304, European Central Bank.

Ngo, P. V. (2019) Fiscal multipliers at the zero lower bound: The role of government spending persistence. Macroeconomic Dynamics, 1-28.

Rotemberg, J. (1982) Monopolistic price adjustment and aggregate output. Review of Economic Studies 49(4), 517-531.

Schmidt, S. (2016) Lack of confidence, the zero lower bound, and the virtue of fiscal rules. Journal of Economic Dynamics and Control 70, 36-53.

Schmitt-Grohé, S. and M. Uribe (2017) Liquidity traps and jobless recoveries. American Economic Journal: Macroeconomics 9(1), 165-204.

Smets, F. and R. Wouters (2007) Shocks and frictions in US business cycles: A bayesian DSGE approach. American Economic Review 97(3), 586-606.

Sugo, T. and K. Ueda (2008) Eliminating a deflationary trap through superinertial interest rate rules. Economics Letters 100(1), 119-122.

Ueda, K. (2001) Costs of inflation in Japan: Tax and resource allocation. Bank of Japan Working Paper Series, Bank of Japan. 